\title{
Design of a Patient-Centered Appointment Scheduling with Artificial Neural Network and Discrete Event Simulation
}

\author{
Wen-Jen Chang ${ }^{1,2^{*}}$, Yen-Hsiang Chang ${ }^{3,4}$ \\ ${ }^{1}$ Department of Information Management, Chang Gung University, Taiwan \\ ${ }^{2}$ Department of General Dentistry, Chang Gung Memorial Hospital, Taiwan \\ ${ }^{3}$ Graduate Institute of Dental and Craniofacial Science, Chang Gung University, Taiwan \\ ${ }^{4}$ Department of General Dentistry, Taoyuan Medical Center, Chang Gung Memorial Hospital, Taiwan \\ Email: *wjchang@mail.cgu.edu.tw, cyh4714@hotmail.com
}

How to cite this paper: Chang, W.-J. and Chang, Y.-H. (2018) Design of a Patient-Centered Appointment Scheduling with Artificial Neural Network and Discrete Event Simulation. Journal of Service Science and Management, 11, 71-82. https://doi.org/10.4236/jssm.2018.111007

Received: December 11, 2017

Accepted: February 10, 2018

Published: February 13, 2018

Copyright $\odot 2018$ by authors and Scientific Research Publishing Inc. This work is licensed under the Creative Commons Attribution International License (CC BY 4.0). http://creativecommons.org/licenses/by/4.0/

\section{Open Access}

\begin{abstract}
Quality of medical services is even more critical than ever in a highly competitive health care industry. Long waiting time is a major source of patient dissatisfaction, thus, the issue of how to reduce waiting time to improve service quality is very important. A well-designed appointment scheduling system can effectively shorten the patient waiting time and enhance their satisfaction. This study aims to propose a framework for individualized outpatient appointment scheduling (OAS) in a dental clinic which composed of one attending dentist and two resident dentists. To design the OAS, firstly, the prediction model of the treatment duration of an individual patient was established by using artificial neural network. Secondly, discrete event simulation method was used to develop the simulation model which simulates the operations of the studied dental clinic. Finally, the established simulation model was used to evaluate the performance of the appointment scheduling. The proposed model consists of numbers of main features: 1) the service providers composed of multiple dentists with different professional competence levels; 2) there are two types of patients (return and not-return patients); 3) patient no-shows was considered; and 4) a variety of medical treatments (requiring different treatment time) are provided to the patients. The results of the study show that the proposed OAS can effectively improve the service performance of the dental clinic, this could be caused by patient's characteristics were taken into consideration of building an appropriated appointment interval.
\end{abstract}

\section{Keywords}

Outpatient Appointment, Appointment Scheduling, Dental Care Services, 
System Simulation, Artificial Neural Network

\section{Introduction}

The trend of health awareness and population aging has led to a surge in demand for health care recently in the developed countries. To provide adequate medical services to cope with the huge increase in the demand of medical treatment, the medical service industry is booming and people have been paying more attention to the quality of medical service. For providing good services to patients, medical institutions are committed to enhance their service quality.

In recent years, outpatient clinics play a more central role in healthcare system [1]. The increasing use of outpatient health care resulted in the expanding of the spending on outpatient services. The proportion of the outpatient service is getting more and more. Outpatient care has become more common due to the introducing of new healthcare technologies, an emphasis on preventive medicine, and to reduce total medical expense. Outpatient visits increased $1.2 \%$ to more than 787 million in 2013, while inpatient admissions declined by $2 \%$ to about 35.4 million, according to annual statistics from the American Hospital Association cited in Modern Healthcare [2]. In Taiwan, the number of visits to the west medical clinics and dental clinic increased by $5.7 \%$ and $18.6 \%$, respectively from 2005 to 2014 [3].

Service quality of outpatient clinics are affected by numerous factors [4] [5], one of which is the design of the outpatient appointment system [6] [7]. Usually, a patient will make an appointment to obtain the scheduled time for his/her clinical visit before the outpatient service, and then he/she will visit the clinic according to this message [8]. However, the medical service system is highly uncertain. For example, the unknown condition of a patient may lead to different treatment durations, a patient may not show up for the scheduled appointment, and the treatment duration for the current patient may be longer than expected, thus delaying the treatment for the next patient and prolonging the waiting time of all the other patients [9]. As a result, the design of the outpatient appointment becomes even more complex. Studies show that delay of clinic treatment and lengthy waiting time are the main causes of negative impact on patient satisfaction [10]. On the other hand, patient no-shows may make a dentist idle, without seeing any patient temporarily and resulting in a waste of resource [11] [12]. A well-designed outpatient appointment system can not only effectively shorten the patient waiting time to reduce congestion and improve patient satisfaction but also increase the utilization of the dentist's service capacity. Therefore, the design of a good outpatient appointment system is one of the most important tasks in effective management of outpatient medical services [13].

In recent years, care for oral health has been gaining more and more attention. Studies have found that oral diseases (dental caries, periodontal disease, 
etc.) are an important risk factor for cardiovascular, diabetes and other diseases [14]. To keep oral diseases from harming someone's health, carrying out daily oral hygiene and engaging dental examinations and professional dental care regularly is necessary for everyone. Therefore, it is important to discuss the design of the outpatient appointment system for dental service. This study explores the problems of outpatient dental services, emphasizing the following characteristics: the service system is composed of multiple service providers (one attending dentist and two resident dentists) with different levels of professional competence and the attending dentist being responsible for the decision of the dental treatments. The objectives of this study is: 1) to develop the rules for outpatient appointments, so as to better arrange the appropriate appointment to reduce patient waiting time and to improve the utilization rate of dentist service capacity; and 2) to establish a simulation model to assist decision makers in assessing the impact of the appointment-making rules.

\section{Problem Description}

The dental clinic discussed in this study offers two types of appointment: open and non-open appointment. In the open appointment, a patient, named new patient, should make an appointment without personal information and will be scheduled his/her own appointment. Usually, the oral health of the open-appointed is unknown to the dentist before the clinic visit. On the other hand, non-open appointment is only provided to return patients, whose next follow-up treatment will be arranged by the dentist according to patients' needs. In this study, we focus on the scheduling design of open dental outpatient appointment.

In the studied dental clinic, patients can make appointments through the system and the appointments are scheduled sequentially and immediately according to the specific scheduling rules. After a patient completes his/her appointment, the system will inform the patient when his/her appointment time is. The outpatient service system is composed of one attending dentist and two resident dentists, meaning that multiple service providers possess different levels of professional competence. In comparison with the literature, the studied service system has two important features: 1) Service providers have different professional competence, where the attending dentist has better expertise than the residents; 2) The service procedure has the re-entry characteristics. Usually, the attending dentist will see the patient first to determine his/her initial conditions and the need for priority treatment. Then, the attending dentist will, as appropriate, assign some of the patients to the resident dentists for follow-up treatment, and finally the attending dentist will check whether the resident dentists have handled the follow-up treatment appropriately before patients leave the clinic.

Patients were classified as new and return. New patients are those who make the appointment through open appointment system or the walk-ins, whose oral health conditions and the required treatments are completely unknown to the 
dentists before visiting. On the other hand, the return patients are follow-up patients with predetermined treatment and are scheduled by the dentist at the last visit. Therefore, the treatment durations of the return patients can be estimated more accurately. The operation of current outpatient appointment system in the studied dental clinic is shown as Figure 1.

The appointment scheduling rule currently used in the studied dental clinic consists in scheduling 4 patients for the beginning of the clinic session followed by 3 to 4 patients each scheduled for a 30-minute block. Time slots are reserved for three walk-ins (mainly retained at the 9:20 - 9:50 interval), and the last patients are scheduled 50 minutes before the end of the outpatient session. In total, 25 patients are scheduled for each outpatient session. To avoid server idle time caused by patient no-shows, a few returning patients are arranged for follow-up visit. Currently, the outpatient appointment system accepts appointments for the next four weeks. When the number of appointments reaches the upper limit, the system will stop accepting any appointment. The outpatient appointment system is usually fully booked at this stage, but patient no-shows often occur, resulting in the negative impact of the service system. Thus, it is absolutely necessary to design an appropriate outpatient appointment scheduling (OAS) for the dental care, so as to enhance the performance of the service system.

In summary, this study investigates the scheduling problem of open dental outpatient appointment. The distinctive features of the problem are the service system comprises multiple service providers with different competence levels and multiple patient flow with the characteristic of re-entry.

\section{Methods}

This study was reviewed and approved by the Institute Review Board (IRB) at the studied hospital, Chang Gung Memorial Hospital. The study population was comprised of the outpatients who visited the Department of General Dentistry at the studied open appointed service system. There are 688 data collected, including the variables of patient age, gender, the length of time since last visit with dentist, the major patient complaints, and the patient's oral care habits, the item of treatments, and the treatment duration. The collected data are used to establish a predicting model for an individual patient's treatment duration and construct a simulation model for reproducing the operating of the studied dental clinic.

In this study, we proposed a framework for outpatient appointment scheduling which consist of three stages. In the first stage, we established an artificial neural network (ANN) model to predict an individual patient's treatment duration for an appropriately appointment time slot. ANN is a renowned and extensively

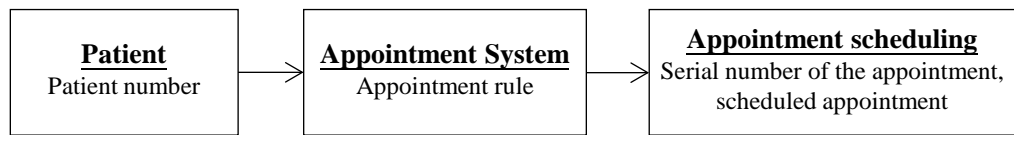

Figure 1. The operation of currently appointment system. 
used for predictions and have been widely employed in numerous fields of science and technology [15]. In the second stage, we developed a simulation model to simulate the operating of the dental clinic. In the third stage, we evaluate the performance of the OAS by using the combination of the constructed simulation model and the established predicted model of ANN.

\subsection{Prediction Model for Individual Treatment Duration}

Treatment durations for dental patients vary greatly from person to person, it's difficult to accurately estimate the treatment durations due to the variety of the treatment for each patient. Therefore, individualized appointment interval is needed. In this study, we constructed an ANN model to predict an individual patient's treatment duration for an appropriately appointment. Back-propagation artificial neural network (BP-ANN) is one of the most representative and most common used model among the artificial neural networks [16] [17]. In general, the architecture of a BP-ANN involves three layers, the first layer represents the input vector that receives information, the last layer is the output that calculates results, and the hidden layer processes information (demonstrate in Figure 2). BP-ANN is featured for its good predictive capability that better than traditional statistical methods [16] and has been widely used in various fields.

To construct the prediction model of an individual's treatment durations, a standard back-propagation algorithm for feed-forward neural networks was applied due to its relative simplicity and stability [17]. The input vector of the BP-ANN consists of the factors that affect the patient's treatment duration and the output vector is the variable being predicted. After analysis, the input vector, named predictors, consists the variables of patient age, gender, the length of time since last visit with dentist, the major patient complaints, and the patient's oral care habits, and the output variable is the patient's treatment duration. The numbers of neurons in the input and output layers was determined by the problem and corresponded to the numbers of predictors and output variables respectively.

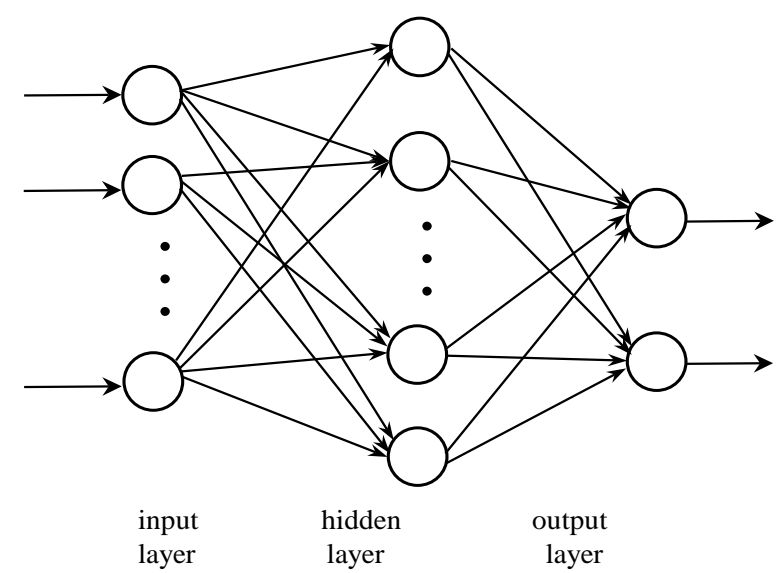

Figure 2. Architecture of back-propagation artificial neural network with one hidden layer. 
The BP-ANN predictive model were established by using the validated data which excluded the collected data with missing value(s). The data were normalized and randomly divided into two groups: training data (70\% of all of the data) and testing data (30\% of all of the data) [18]. To construct a BP-ANN, all data were normalized to the range of 0 to 1 . For the binary variables, 0 represents "no" and 1 means "yes". For a nonbinary variable $(\mathrm{X})$ was normalized as the formula: $x_{i}^{\prime}=\left(x_{i}-x_{\min }\right) /\left(x_{\max }-x_{\min }\right)$, where $x_{\min }$ and $x_{\max }$ denote the minimum and maximum value in $\mathrm{X}$, respectively; $x_{i}$ and $x_{i}^{\prime}$ denote the value of real observation and normalization of the $i$ th variable, respectively.

For network training, sigmoid functions were used as the transfer functions of the hidden and output layers. The gradient descent algorithm was used as an adaptive learning function. The optimum network of the BP-ANN is usually determined by carrying out an experimentation of trial-and-error. [15]. The trial rage of the number of hidden neurons $(H)$ could be determined as follow: $\boldsymbol{H}=\sqrt{\boldsymbol{I}+\boldsymbol{O}}+c$, where $\boldsymbol{I}$ and $\boldsymbol{O}$ is the number of neurons in the input and output layers respectively; $c$ is a constant ranging from 0 to 10 [17]. We changed the number of hidden neurons from three to twelve, increased 0.05 of the learning rate from 0.1 to 0.5 . The ANN efficiency is evaluated using the mean squared error (MSE). The networks were trained at a maximum of 250 epochs or the mean square error was less than 0.0025. The MSE is defined as Equation (1), where $\mathrm{n}$ is the number of data points, $T_{i}$ is the real treatment duration of patient $i$, and $\tilde{T}_{i}$ is the predicted treatment duration of patient computed by the artificial neural network:

$$
\operatorname{MSE}=\frac{\sum\left(T_{i}-\tilde{T}_{i}\right)^{2}}{n}
$$

\subsection{Simulation Model}

Operating of the dental clinic was simulated by constructing the simulation model. Discrete event simulation (DES) method is used to establish the outpatient treatment process of the dental clinic and a version 7.5 of Flexsim software was used. DES technique is wide use to model the operations of a system where entities compete for limited resources, forming queues as needed [19]. For the assessment of health technology, a flexible event based DES model can address a wide variety of the problem [20] [21].

In this study, two simulation models, namely real model and theoretical model, was created. The real model attempts to reproduce the current operating process at the clinic. The theoretical model aims to evaluate the performance of the appointment scheduling.

To illustrate the actual operation of the clinic, the real model was built and tested to make sure that is represented the collected real data. Patient arrival time and the end time of the treatment was considered in the real model. The accuracy of the model is verified by the difference of the average cycle time between actual observation and simulation (Cycle time refers to the interval be- 
tween the arrival time and the end time of the treatment). Researches indicate that cycle time is a good indicator of the accuracy of the real model because it includes all the independent variables (e.g., waiting time, treatment duration, arrival rate, etc.) [22]. In the simulation process, we take three clinic sessions from the observation records to verify the accuracy of the real model and execute 30 replications per clinic sessions. The results of the average cycle time of the actual observation and simulation was shown in Table 1 . The difference of average cycle time between the actual observation and the simulation in each simulated clinic session is less than $5 \%$, so that the developed simulation model is accurate enough to represent the actual operation of the clinic.

The theoretical model was developed in the bases of real model, therefore, the two model are similar the only exception is that patient arrival time in the theoretical model is determined by the appointment scheduling under the assuming of patients arriving on time. To accurately measure the performance of OAS, the patient being late or early arrival is not considered in the theoretical model. The performance evaluation of the OAS was demonstrated in the next section.

\subsection{Outpatient Appointment Scheduling}

An individual treatment duration can be predicted by the established BP-ANN model and was use to set an appointment interval with appropriate adjustment. The appointment interval was derived from the predicted treatment duration divided by the patient attendance rate. Patient no-show will result in the doctor being idle, and the waste of the service capacity. Accordingly, we shortened the interval of the appointment to reduce the negative impact of patient no show.

For a clinic session, patient was appointed sequentially according the adjusted appointment interval. The $k$ th patient's appointment time is the sum of the previous $(k-1)$ patients' appointment intervals, that is $\sum_{i=1}^{k-1}\left(T_{i} / r\right)$ after the begging of the clinic opened, where $T_{i}$ denotes the predicted treatment duration of $i$ th patient, and $r$ means the patient attendance rate. The attendance rate were derived from the collected data. Except for the appointed patient, a clinic session provide services for two return patients and up to three walk-in patients as currently. The return patients with predetermined follow-up treatment will be arranged at the beginning of a clinic session. According to the collected data there are on average only one walk-ins every clinic session. Inter-arrival of walk-ins were determined from real walk-ins arrival times. As the result, the outpatient appointment scheduling is derived and the proposed model is illustrated in Figure 3.

Table 1. The comparison of average cycle time of observed and simulated data.

\begin{tabular}{cccc}
\hline & $\begin{array}{c}\text { Average observed } \\
\text { cycle time }(\mathrm{min})\end{array}$ & $\begin{array}{c}\text { Average simulated } \\
\text { cycle time }(\mathrm{min})\end{array}$ & Difference (\%) \\
\hline Day 1 & 43.05 & 43.95 & $2.09 \%$ \\
Day 2 & 37.35 & 35.85 & $4.18 \%$ \\
Day 3 & 52.63 & 54.82 & $4.15 \%$ \\
\hline
\end{tabular}




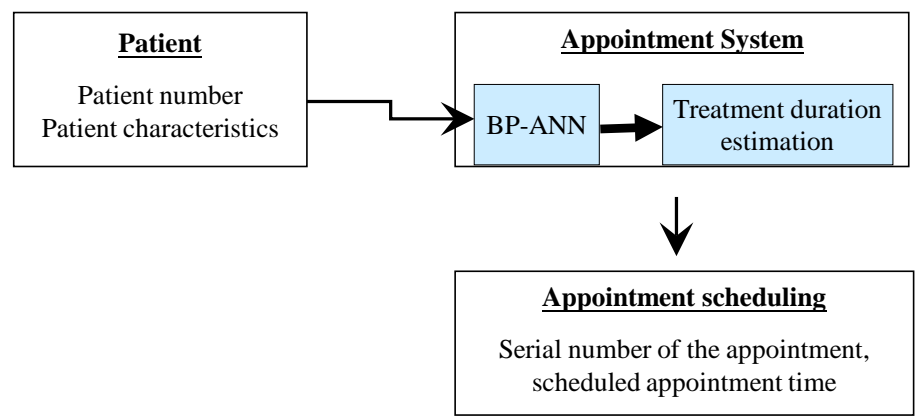

Figure 3. The proposed model for the appointment scheduling model.

The performance of the OAS was evaluated by the theoretical model. To measure the performance of OAS accurately, patient arrivals are determined according to the scheduled appointments. That is, we assume patients arrive on time. The performance assessment of the OAS consists three indices: cycle time, the number of patients seen, and the utilization of the service capacity. A good OAS will result in short the cycle time, large number of patients seen, and high utilization of the service capacity.

\section{Results and Discussion}

\subsection{The Results}

Initially, there were 688 data collected, and 58 observations with missing values were excluded from the study. Of the 630 observations, 298 (47.3\%) were men, and 332 were women (52.7\%). The patients are raged $44.6 \pm 17.6$ years old (mean \pm standard deviation). The highest number of patients' complaints related to the regular dental exams (39.7\%), and toothache (38.7\%). The length of time since last visit with dentist of most patients are between one to two years (31.8\%).

For establishing BP-ANN, the 630 validated data were randomly divided into a training set with 450 observations and a testing set with 180 observations (about $70 \% / 30 \%$ of training/testing data). The input and output layers contain 5 and 1 neuron, respectively, which corresponding to the predictors and output variables, respectively. The input variables are the predictors: patient age, gender, the length of time since last visit with dentist, the major patient complaints, and the patient's oral care habits. The output variable is the patient's treatment duration. The number of neuron in the hidden layer were tested by using the aforementioned equation ranges from 3 to 12 . The value of learning rate and momentum were set as 0.15 and 0.8 respectively. Finally, we obtained three layers of back-propagation neural network architecture, which consists 5 nodes in the input layer, 1 node in the output layer, and 7 nodes in the hidden layer. The accuracies of the established BP-ANN on training and testing datasets are $90.2 \%$ and $85.5 \%$, respectively. Thus, a well-trained BP-ANN is derived and could effectively predict the treatment duration of an individual patient.

In the outpatient delivery system, patient no-shows will result in a waste of 
service capacity. Currently the average rate of no-shows is $26.8 \%$. To avoid the negative impact of no-shows on the service system, the appointment intervals should be adjusted. A patient's appointment interval is derived from dividing the predicted treatment duration by the patient attendance rate. Patients were scheduled

The appointment time of a patient is scheduled sequentially. The $k$ th patient's appointment time is the sum of the previous $(k-1)$ patients' appointment intervals after the begging of the clinic opened. The OAS consists of two types of patients, new and return patients. The performance metrics of OAS consists of three indicators, namely waiting time, utilization of the service capacity, and clinical session duration. Waiting time refers to the time that a patient waits in the clinic before being seen by the dentist. The utilization of the service capacity measured by the index of average proportion of occupied time for dentists. Clinical session duration refers to the interval between the session opening and the time when the last patient leaves the clinic.

\subsection{Comparison and Discussion}

To compare the performance of the outpatient appointment system of the proposed model and that of the current used model, the test for differences between means was used. The results are shown in Table 2 indicate that there is a significant difference between the two scheduling model ( $p$-value $<0.01)$. The performance of the appointment scheduling model proposed in this study is significantly better in all the three indicators. The patient waiting time has been significantly reduced from 35.23 minutes to 24.91 minutes with a reduction by more than 10 minutes (29.3\%). Compare to the literature [10], the average waiting time in the proposed model is less than 30 minutes indicating that the patient is acceptable.

The utilization of the service capacity increases from $82.1 \%$ to $88.5 \%$. Regarding the clinical session duration is considered ideal by administrators to fall within the range of 210 to 240 minutes, the appointment scheduling model proposed by this study shows that the clinical session duration is approximately 226.3 minutes, a reduction about $10 \%$ from 251.4 minutes under currently appointment scheduling model adopted by the clinic.

From the comparison of the three performance indicators described above, it can be found that the proposed outpatient appointment scheduling can perform significantly better than the clinic's current model. There may be three main

Table 2. Comparison of the performance obtained by current used and proposed appointment scheduling model.

\begin{tabular}{cccc}
\hline & $\begin{array}{c}\text { Patient waiting time } \\
(\mathrm{min})\end{array}$ & $\begin{array}{c}\text { Proportion of occupied } \\
\text { time for dentist }(\%)\end{array}$ & $\begin{array}{c}\text { Session duration } \\
(\mathrm{min})\end{array}$ \\
\hline Currently used & 35.23 & 82.08 & 241.51 \\
Proposed model & 24.91 & 88.05 & 226.33 \\
p-value & 0.003 & $<0.001$ & 0.003 \\
\hline
\end{tabular}


reasons when the differences between the two models are compared:

1) The proposed appointment model can schedule patient more appropriately: As the current method of outpatient appointment does not collect information on the patient characteristics, and the required treatment durations for patients are estimated on the basis of "average" concept. However, the treatment durations for dental visits vary greatly, resulting in difficulties in scheduling the appointments and poor performance of the service system. In contrast, the proposed appointment model requires patients to provide their own patient characteristics at the time of appointment making. This design enables our model to estimate the treatment duration more accurate for individual patients, thereby significantly improving the performance of the outpatient service system.

2) Number of appointments in the initial block: To avoid patient no-shows in the initial block from causing idle service capacity, the proposed model puts 4 appointments in the initial block, two of which is reserved for a return patient, thus improving the utilization of dentist's service capacity.

3) Planning for the walk-ins: according to the collected data, the actual number of walk-ins has almost never reached the number of current reservations (Three appointments are reserved for walk-ins but actually the average walk-in is only one). Therefore, we suggest that the dental clinic still provide up to three walk-in patients without reserving a specific appointment time slot, and the walk-ins be admitted depending on the actual status of the clinic, so as to avoid idle service capacity.

\section{Conclusions}

We investigate a complex outpatient appointment scheduling problem in dental clinic which comprises multiple service providers with different competence levels and multiple patient flow with the characteristic of re-entry. In this study, an artificial neural network based model is constructed for predicting the patients' treatment duration so as to set up rules for individual patients to make an appointment and to find the best appointment interval. Finally, a simulation model is used to evaluate the performance of outpatient appointment scheduling. The dynamics and randomness of the service system, such as walk-ins and no-show rate, is considered in the simulation model.

The proposed appointment scheduling model can significantly improve the performance of outpatient services of the studied dental clinic. The main contribution of this study is developing a patient-centered appointment time slot in order to enhance the performance of the outpatient service system. The treatment duration depend on patient characteristics and vary greatly. In this study, we construct an artificial neural network model to predict the treatment durations for individual patients to make a good appointment scheduling.

\section{Acknowledgements}

This research was supported by grant MOST105-2221-E-182-045 from the Min- 
istry of Science and Technology, Taiwan.

\section{References}

[1] Ahmadi-Javid, A., Jalali, Z. and Klassen, K.J. (2017) Outpatient Appointment Systems in Healthcare: A Review of optimization Studies. European Journal of Operational Research, 258, 3-34. https://doi.org/10.1016/j.ejor.2016.06.064

[2] Robeznieks, A. (2015) Hospitals Saw Fewer Admissions, More Outpatients in 2013. Modern Healthcare. http://www.modernhealthcare.com/article/20150127/NEWS/301279903

[3] Ministry of Health and Welfare, Executive Yuan (2015) Health Statistics in Taiwan. https://www.mohw.gov.tw/dl-18872-0cf3d73a-c867-4c52-85da-7ea006861bd5.html

[4] Harper, P.R. and Gamlin H.M. (2003) Reduced Outpatient Waiting Times with Improved Appointment Scheduling: A Simulation Modelling Approach. OR spectrum, 25, 207-222. https://doi.org/10.1007/s00291-003-0122-x

[5] Chung, K. and Min, D. (2014) Staffing a Service System with Appointment-Based Customer Arrivals. Journal of the Operational Research Society, 65, 1533-1543. https://link.springer.com/article/10.1057/jors.2013.110

[6] Klassen, K.J. and Yoogalingam, R. (2009) Improving Performance in Outpatient Appointment Services with a Simulation Optimization Approach. Production and Operations Management, 18, 447-458.

https://doi.org/10.1111/j.1937-5956.2009.01021.x

[7] Pan, C., Zhang, D., Kon, A.W., Wai, C.S. and Ang, W.B. (2015) Patient Flow Improvement for an Ophthalmic Specialist Outpatient Clinic with Aid of Discrete Event Simulation and Design of Experiment. Health Care Management Science, 18, 137-155. https://doi.org/10.1007/s10729-014-9291-1

[8] Zhao, P., Yoo, I., Lavoie, J., Lavoie, B.J. and Simoes, E. (2017) Web-Based Medical Appointment Systems: A Systematic Review. Journal of Medical Internet Research, 19, e134. https://doi.org/10.2196/jmir.6747

[9] Cao, W., Wan, Y., Tu, H., Shang, F., Liu, D., Tan, Z., et al. (2011) A Web-Based Appointment System to Reduce Waiting for Outpatients: A Retrospective Study. BMC Health Services Research, 11, 318. ttps://doi.org/10.1186/1472-6963-11-318

[10] Zarei, E. (2015) Service Quality of Hospital Outpatient Departments: Patients' Perspective. International Journal of Health Care Quality Assurance, 28, 778-790. https://doi.org/10.1108/IJHCQA-09-2014-0097

[11] Chang, W.J. and Chang, Y.H. (2013) Patient Satisfaction Analysis: Identifying Key Drivers and Enhancing Service Quality of Dental Care. Journal of Dental Sciences, 8, 239-247. https://doi.org/10.1016/j.jds.2012.10.006

[12] Zacharias, C. and Pinedo, M. (2014) Appointment Scheduling with No-Shows and Overbooking. Production and Operations Management, 23, 788-801.

https://doi.org/10.1111/poms.12065

[13] El-Sharo, M., Zheng, B., Yoon, S.W. and Khasawneh, M.T. (2015) An Overbooking Scheduling Model for Outpatient Appointments in a Multi-Provider Clinic. Operations Research for Health Care, 6, 1-10. https://doi.org/10.1016/j.orhc.2015.05.004

[14] Cayirli, T., Veral, E. and Rosen, H. (2008) Assessment of Patient Classification in Appointment System Design. Production and Operations Management, 17, 338-353.

[15] Raghupathi, V. and Raghupathi, W. (2017) Preventive Healthcare: A Neural Net- 
work Analysis of Behavioral Habits and Chronic Diseases. Healthcare, 5, 8. https://doi.org/10.3390/healthcare5010008

[16] Lamster, I.B., Lalla, E., Borgnakke, W.S. and Taylor, G.W. (2008) The Relationship between Oral Health and Diabetes Mellitus. The Journal of the American Dental Association, 139, 19S-24S. https://doi.org/10.14219/jada.archive.2008.0363

[17] Carlucci, D., Renna, P. and Schiuma, G. (2013) Evaluating Service Quality Dimensions as Antecedents to Outpatient Satisfaction Using Back Propagation Neural Network. Health Care Management Science, 16, 37-44. https://doi.org/10.1007/s10729-012-9211-1

[18] Vellido, A. Lisboa, P.J.G. and Vaughan, J. (1999) Neural Networks in Business: A Survey of Applications (1992-1998). Expert Systems with Applications, 17, 51-70. https://doi.org/10.1016/S0957-4174(99)00016-0

[19] Caro, J.J., Möller, J., Karnon, J., Stahl, J. and Ishak, J. (2015) Discrete Event Simulation for Health Technology Assessment. CRC Press, Taylor \& Francis Group, Boca Raton (FL). https://doi.org/10.1201/b19421

[20] Caro, J.J. and Möller, J. (2016) Advantages and Disadvantages of Discrete-Event Simulation for Health Economic Analyses. Expert Review of Pharmacoeconomics \& Outcomes Research, 16, 327-329. https://doi.org/10.1586/14737167.2016.1165608

[21] Aeenparast, A., Tabibi, S.J., Shahanaghi, K. and Aryanejhad M.B. (2013) Reducing Outpatient Waiting Time: A Simulation Modeling Approach. Iranian Red Crescent Medical Journal, 15, 865-869. https://doi.org/10.5812/ircmj.7908

[22] Baril, C., Gascon, V. and Cartier, S. (2014) Design and Analysis of an Outpatient Orthopaedic Clinic Performance with Discrete Event Simulation and Design of Experiments. Computers \&Industrial Engineering, 78, 285-298.

https://doi.org/10.1016/j.cie.2014.05.006 